\title{
Pseudotensor mesons as three-body resonances
}

\author{
L. Roca \\ Departamento de Física. Universidad de Murcia. E-30071, Murcia. Spain
}

(Dated: October 30, 2018)

\begin{abstract}
We show that the lightest pseudotensor mesons $J^{P C}=2^{-+}$can be regarded as molecules made of a pseudoscalar $(P) 0^{-+}$and a tensor $2^{++}$meson, where the latter is itself made of two vector $(V)$ mesons. The idea stems from the fact that the vector-vector interaction in s-wave and spin 2 is very strong, to the point of generating the $2^{++}$tensor mesons. On the other hand the interaction of a pseudoscalar with a vector meson in s-wave is also very strong and it generates dynamically the lightest axial-vector mesons. Therefore we expect the $P V V$ interaction to be strongly attractive and thus able to build up quasibound $P V V$ resonances. We calculate the three body $P V V$ interaction by using the fixed center approximation to the Faddeev equations where the two vectors are clustered forming a tensor meson. We find clear resonant structures which can be identified with the $\pi_{2}(1670)$, $\eta_{2}(1645)$ and $K_{2}^{*}(1770)\left(2^{-+}\right)$pseudotensor mesons.
\end{abstract}

\section{INTRODUCTION}

Unveiling the structure and nature of hadrons is of crucial importance to understand the strong interaction. Several different components can contribute to the wave functions of the mesonic resonances besides the simple quark-antiquark state. In many mesons the quarkantiquark component is the dominant one. However, for some specific mesonic resonances, other contributions such as glueballs, tetraquarks and meson molecules can dominate their wave function. If the meson-meson interaction is attractive the meson molecule component may be dominant and the dynamical generation of the resonances may be more efficient than the other Fock space terms. In the present work, this will be a recurrent idea for many resonances considered. In the mesonic sector, important results regarding the molecular interpretation have been obtained by the unitary extensions of chiral perturbation theory (UChPT or chiral unitary approach). Using as input lowest orders chiral Lagrangians and implementing unitarity in coupled channels, many resonances are obtained from the meson-meson or mesonbaryon interaction [1 11], which are also usually called dynamically generated resonances.

In particular, it is of special interest for the present work the pseudoscalar-vector and vector-vector unitarized interaction. In the last few years several works 12 15] have reported arguments and evidence for a dynamical nature of the lightest axialvector resonances, implementing variants of the chiral unitary approach. The axialvector resonances naturally appear [12, 13] as poles in the scattering matrix of the interaction of pseudoscalar mesons with vector mesons. Therefore most of the lowlying axialvector mesons can be described by dynamics of a pseudoscalar and a vector meson and thus can be regarded as molecules made of a pseudoscalar and a vector meson.

On the other hand, the vector-vector interaction has been recently studied [16 18] using the techniques of the chiral unitary approach, using as input for the vectorvector potential the lowest order hidden gauge symmetry Lagrangian [19 22]. In [17] eleven resonant states were found in nine strangeness-isospin-spin channels. In particular, and of interest for the present work, the lightest tensor $2^{++}$mesons $a_{2}(1320), f_{2}(1270)$ and $K_{2}^{*}(1430)$ were dynamically generated from the $V V$ interaction in s-wave and spin two and they where found to be dominantly molecules made of $K^{*} \bar{K}^{*}, \rho \rho$ and $\rho K^{*}$ respectively 1 .

From the previous considerations, it is reasonable to expect that a system made of a pseudoscalar and two vector mesons $(P V V)$ is bound given the strong attraction between the two vector mesons in s-wave with parallel spins (which in turn form a tensor meson) and the strong interaction of the pseudoscalar with the two constituent vector mesons. These possible bound (or quasibound) states would have $J^{P C}=2^{-+}$quantum numbers which could correspond to some known (or still undiscovered) pseudotensor resonances. The main aim of the present work is to carry out the theoretical study of such possibility. Indeed, and bringing forward some results of this work, we will find several $P V V$ resonant structures which may be associated to the $\pi_{2}(1670), \eta_{2}(1645)$ and $K_{2}^{*}(1770)$ resonances.

The idea of the existence of three body resonances is of course not new. However, while much work has been done in the baryonic sector, (e.g. [24 27]), less studies have been devoted to three meson molecules [28 30]. The proper analysis of the three body problem, like the one required in the present work to study the $P V V$ interaction, can be conceptually tackled by the implementation of the Faddeev equations [31]. However they are very difficult to solve exactly, hardly ever possible, and almost always one has to recur to approximations. For a recent fresh look into the problem see Ref. [26] for two mesonone baryon systems and [29, 230, 32] for three mesons.

\footnotetext{
${ }^{1}$ Actually, in Ref. [17] the pole in $K^{*} \bar{K}^{*}$ amplitude was not associated to the $a_{2}(1320)$ resonance since it was far from the experimental mass. However we discuss in the present work that the $a_{2}(1320)$ can indeed be found in this channel with a slight modification of the only free parameter of Ref. [17], as pointed out in ref [23].
} 
When two of the three particles are bound forming a cluster, as will be the case in the present work, one can use the fixed center approximation (FCA) to the Faddeev equations [33 37]. When applicable, the idea is very simple and considers that one particle collides against the two particles of the cluster which is not much altered by the collision, which requires energies close or below threshold [38]. Recently, the FCA has been successfully applied in many three body interactions 36, 39 43. In the present work we apply the FCA to the Faddeev equations to evaluate the interaction of a pseudoscalar meson with two vector mesons in spin 2 and s-wave, where the vector mesons are bound making up a tensor meson.

\section{TWO-BODY INTERACTIONS}

In the system that we consider in the present work, one pseudoscalar $(P)$ and two vector $(V)$ mesons, one of the most important ingredients are the two-body interactions, $V V$ and $P V$. The $V V$ interaction is needed in order to show that the $V V$ amplitudes in s-wave and spin 2 is very attractive, which generate dynamically the lightest $2^{++}$tensor mesons, and to know to which particular $V V$ channel each tensor resonance couples most.

On the other hand, the $P V$ amplitudes are needed in the FCA equations since we will write the three-body scattering amplitudes in terms of the two-body interaction of the pseudoscalar meson with each of the two particles in the cluster. We summarize in what follows the models for the $V V[16,17]$ and $P V$ [13] unitarized interaction properly adapted to the present work.

\section{A. Vector-vector unitarization}

The model of Refs. [16, 17] applies the ideas of the chiral unitary approach to the evaluation of the $V V$ scattering amplitudes. The implementation of unitarity in coupled channels and the exploitation of the analytic properties of the scattering amplitudes leads to the full twobody scattering amplitude for a given partial wave, which can be written by means of the Bethe-Salpeter equation in coupled channels in the following way:

$$
t=V+V G t=(1-V G)^{-1} V
$$

where the kernel $V$ is a matrix containing the elementary vector-vector transition amplitudes and $G$ is a diagonal matrix with the $l^{\text {th }}$ element, $G_{l}$, given by the loop function for two vector mesons:

$$
G_{l}=i \int \frac{d^{4} q}{(2 \pi)^{4}} \frac{1}{(P-q)^{2}-M_{l, V 1}^{2}} \frac{1}{q^{2}-M_{l, V 2}^{2}}
$$

where $P$ is the total four-momentum of the $V V$ system and $M_{l, V 1}$ and $M_{l, V 2}$ are the masses of the two vectormesons of the corresponding $l^{\text {th }}$ channel. In the loop function, the widths of the vector mesons are accounted for by folding Eq. (2) with their spectral functions as explained in Ref. [17].

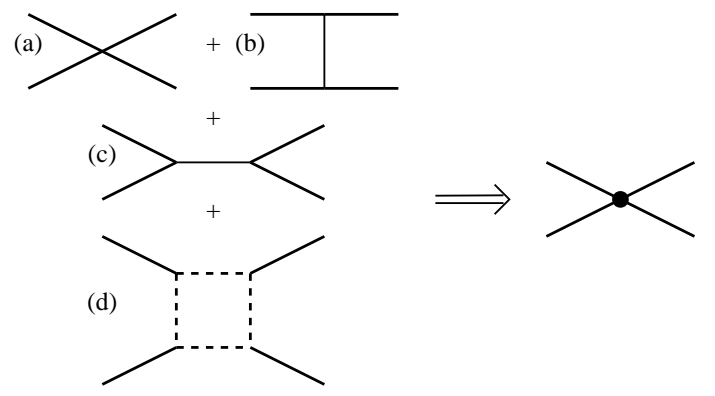

FIG. 1. Mechanisms contributing to the kernel $V$ (thick dot) of the Bethe-Salpeter equation, Eq. (1), for vector-vector scattering. Solid lines represent vector mesons and dashed lines pseudoscalar ones.

The mechanisms contributing to the vector-vector potential $V$, the kernel of the Bethe-Salpeter equation (11), are depicted in Fig. 1, The full kernel $V$ is represented by a thick dot in Fig. 11 to which the mechanisms $(a)$, $(b),(c)$ and $(d)$ provide different contributions. In this figure the solid lines represent vector mesons and the dashed lines pseudoscalar ones. For the evaluation of these diagrams we need the 4 -vectors, 3 -vectors and one vector-2-pseudoscalars vertices which are obtained from the hidden gauge symmetry Lagrangian [19 22] for vector mesons. Explicit expressions for the Lagrangians and the $V$-matrix elements for the different channels can be found in Refs. [16, 17, 39]. The dominant contribution to the potential comes from the contact term, Fig. 11(a), and the $t, u$ channel exchange, Fig. 11(b). The s-channel, Fig. 1(c), is very small since it is basically p-wave. The box diagram, Fig. 1(d), is relevant only for the width of the generated resonance [16, 17] and it allows the decay into two pseudoscalar mesons.

The previous formalism can be applied to any possible strangeness-isospin-spin channel but we are interested in the present work in the spin 2 channel in swave. In the modulus squared of the different scattering amplitudes, prominent resonant shapes appear (we refer to Ref. [17] for explicit plots) which also correspond to poles in unphysical Riemann sheets in the complex energy plane, $\sqrt{s}$. For spin 2 there are three possible channels. The first one is strangeness 0 and isospin 1 to which $K^{*} \bar{K}^{*}, \rho \rho, \phi \phi, \omega \omega$ and $\omega \phi$ contribute. A pole was found at $\sqrt{s}=(1275-1 i)$ which clearly corresponds to the $f_{2}(1270)$ resonance. By evaluating the residues of the scattering amplitudes at the pole position, the couplings of the dynamically generated $f_{2}(1270)$ resonance to the different channels can be obtained. The coupling to $\rho \rho$ is by far the strongest one [17]. This is one of the reasons why the $f_{2}(1270)$ resonance can be considered a $\rho \rho$ molecule or, in other words, a dynamically generated state from $\rho \rho$ interaction. Another of the possible $V V$ 
spin 2 channels is the strangeness 1 , isospin $1 / 2$, to which $K^{*} \rho, K^{*} \omega$ and $K^{*} \phi$ contribute in coupled channels. The unitarized amplitude in this case shows up a resonant shape and a pole at $\sqrt{s}=(1431-i 1)$ which corresponds to the $K_{2}^{*}(1430)$ resonance. In this case, the largest coupling (by a factor 4 ) is to $K^{*} \rho$ channel. Therefore we will consider in the present work the $K_{2}^{*}(1430)$ as a quasibound state of $K^{*} \rho$ interaction. Finally, another $V V$ channel is possible: strangeness 0 and isospin 1 . For this channel the $K^{*} \bar{K}^{*}, \rho \rho, \rho \omega$ and $\rho \phi$ channels are allowed. In Ref. [17] a pole was found at $\sqrt{s}=(1519-i 16)$ with the strongest coupling to $K^{*} \bar{K}^{*}$. The authors of that reference could not clearly assign this pole to any experimental $a_{2}$ resonance. However we are going to argue that this channel can produce the $a_{2}(1320)$ by doing a fine tunning of the only free parameter in the model, which is the regulator parameter of the $V V$ loop functions of Eq. (2). The loop function in Eq. (2) needs to be regularized and this can be accomplished either with a three-momentum cutoff or with dimensional regularization. The equivalence of both methods for meson-meson scattering was shown in Ref. [3]. In Ref. 17] the regularization method was used with subtraction constants, $a$, around a natural value of -1.65 , which corresponds to using a three momentum cutoff of $1 \mathrm{GeV}$. With this natural value the bulk of the resonances appear but a slight fine tuning can be done to agree better with the experimental masses of the $f_{2}(1270)$ and $K_{2}^{*}(1430)$ (see Ref. [17] for the specific values of the subtraction constants used in the original work), but it is worth mentioning that it only provides a slight modification in the position of the peaks. We have checked that using the cutoff regularization the peaks of the $f_{2}(1270)$ and $K_{2}^{*}(1430)$ are reproduced using three momentum cutoffs of $875 \mathrm{MeV}$ for the isospin 0 channel and 972 for the isospin $1 / 2$ channel. Coming back to the isospin 1 channel we can produce a peak in the $V V$ amplitude at the position of the $a_{2}(1320)$ experimental mass using a three momentum cutoff of $1590 \mathrm{MeV}$. Therefore we can consider the $a_{2}(1320)$ as a $K^{*} \bar{K}^{*}$ molecule. The values of the cutoffs described so far will also play a role in the FCA equations later on in the evaluation of the tensor form factors.

In summary, in the later evaluation the $P V V$ interaction we will regard the $f_{2}(1270)$ as a cluster made of $\rho \rho$, the $K_{2}^{*}(1430)$ as $K^{*} \rho$ and the $a_{2}(1320)$ as $K^{*} \bar{K}^{*}$.

\section{B. Pseudoscalar-vector unitarization}

The explicit $P V$ unitarized amplitudes are of crucial importance in the evaluation of the $P V V$ interaction, since we will need to know the interaction of the pseudoscalar meson with each of the two vector mesons. The $P V$ amplitudes we use in the present work are essentially based on the model of Ref. [13], where most of the lightest axialvector resonances were dynamically generated from the interaction of a vector and a pseudoscalar meson. With the only input of the lowest-order chiral La- grangian and the implementation of unitarity in coupled channels the axialvector resonances manifest themselves as poles in unphysical Riemann sheets of the $P V$ scattering amplitudes.

Considering the vector mesons as fields transforming homogeneously under the nonlinear realization of chiral symmetry [44], the interaction of two vector and two pseudoscalar mesons at lowest order in the pseudoscalar fields can be obtained from the following interaction Lagrangian [45]:

$$
\mathcal{L}=-\frac{1}{4}\left\{\left(\nabla_{\mu} V_{\nu}-\nabla_{\nu} V_{\mu}\right)\left(\nabla^{\mu} V^{\nu}-\nabla_{\mu} V_{\nu}\right)\right\},
$$

which is invariant under chiral transformations $S U(3)_{L} \otimes$ $S U(3)_{R}$. In Eq. (3) $\nabla_{\mu} V_{\nu}=\partial_{\mu} V_{\nu}+\left[\Gamma_{\mu}, V_{\nu}\right]$ is the $S U(3)-$ matrix valued covariant derivative, with the $S U(3)$ connection defined as $\Gamma_{\mu}=\left(u^{\dagger} \partial_{\mu} u+u \partial_{\mu} u^{\dagger}\right) / 2, u=$ $\exp (P / \sqrt{2} f)$ and $P$ and $V$ are $S U(3)$ matrices containing the pseudoscalar and vector fields respectively.

From this Lagrangian, the $V P \rightarrow V P$ tree level amplitudes can be obtained expanding Eq. (3) up to two vector and two pseudoscalar meson fields: [12, 13]

$$
\mathcal{L}_{V P}=-\frac{1}{4 f^{2}}\left\langle\left[V^{\mu}, \partial^{\nu} V_{\mu}\right]\left[P, \partial_{\mu} P\right]\right\rangle,
$$

where \langle\rangle stands for $S U(3)$ trace. The explicit expression of the potentials, properly projected onto $s$-wave, is thus

$$
\begin{aligned}
V_{i j}(s)= & -\frac{1}{8 f^{2}} C_{i j}\left[3 s-\left(M_{i}^{2}+m_{i}^{2}+M_{j}^{2}+m_{j}^{2}\right)\right. \\
& \left.-\frac{1}{s}\left(M_{i}^{2}-m_{i}^{2}\right)\left(M_{j}^{2}-m_{j}^{2}\right)\right],
\end{aligned}
$$

where $f=92 \mathrm{MeV}$ is the pion decay constant, the index $i(j)$ represents the initial (final) $V P$ state in the isospin basis and $M_{i}\left(M_{j}\right)$ and $m_{i}\left(m_{j}\right)$ correspond to the masses of the initial (final) vector mesons and initial (final) pseudoscalar mesons, for which we use an average value for each isospin multiplet. In Eq. (5) we have omitted an $\epsilon_{i} \cdot \epsilon_{j}$ term for the polarization of the vector mesons which factorizes. The explicit values of the numerical coefficients, $C_{i j}$, can be found in Ref. [13].

Following the ideas of the chiral unitary approach the full $P V T$-matrix can now be obtained by unitarizing the previous tree level amplitudes which in this case leads to the following Bethe-Salpeter equation:

$$
T=-[1+V G]^{-1} V
$$

which can be diagrammatically represented by the resummation series shown in Fig. 2

Analogously to Eq. (2), $G$ is a diagonal matrix but now with the $l^{\text {th }}$ element, $G_{l}$, given by the loop function of a pseudoscalar and a vector meson,

$$
G_{l}(P)=i \int \frac{d^{4} q}{(2 \pi)^{4}} \frac{1}{(P-q)^{2}-M_{l}^{2}+i \epsilon} \frac{1}{q^{2}-m_{l}^{2}+i \epsilon},
$$




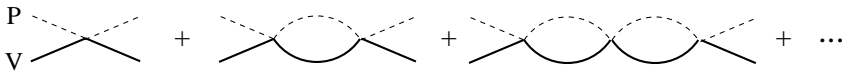

FIG. 2. Diagrammatic interpretation of the unitarization of the $V P \rightarrow V P$ amplitude.

where $P$ is the total four-momentum, $P^{2}=s$, of the $V P$ system. In order to take into account the width of the vector mesons, we fold Eq. (7) by the corresponding vector spectral function 14.

There are nine different possible channels characterized by their strangeness $(S)$, isospin $(I)$ and $G$-parity $(G)$, but not all them have resonant poles in unphysical Riemann sheets of the complex energy plane, i.e. do not generate dynamically resonances. The channels that manifest resonant poles are $(S, I, G)=(0,0,+)$, for which $K^{*} K(+)$ is possible; $(S, I, G)=(0,0,-)$ for which $\phi \eta, \omega \eta, \rho \pi$ and $K^{*} K(-)$ are allowed; $(S, I, G)=$ $(0,1,+)$ with $K^{*} K(+), \phi \pi, \omega \pi, \rho \eta$ as allowed channels; $(S, I, G)=(0,1,-)$ with $\rho \pi$ and $K^{*} K(-)$ and $(S, I)=(1,1 / 2)$ where $\phi K, \omega K, \rho K, K^{*} \eta$ and $K^{*} \pi$ channels are allowed. In the above paragraph $K^{*} K( \pm)$ represent the $G$-parity eigenstates $21 / \sqrt{2}\left(\left|\bar{K}^{*} K\right\rangle \pm\left|K^{*} \bar{K}\right\rangle\right)$ with eigenvalues \pm 1 .

In Ref. [13] seven poles were found in the unphysical Riemann sheets of the unitarized scattering amplitudes which can be associated to most of the lightest axialvector resonances quoted in the Particle Data Group tables (PDG): $b_{1}(1235), h_{1}(1170), h_{1}(1380), a_{1}(1260)$, $f_{1}(1285)$ and the $K_{1}(1270)$ resonance. Actually two poles are present in the unitarized $P V$ amplitude for the $K_{1}(1270)$ resonance. This double pole structure was studied in Ref. [14]. In Ref. [13] all these resonances were obtained using a single value of the parameter needed to regularize the $P V$ loop function, Eq. (7). This parameter was a subtraction constant $a=-1.85$ for the dimensional regularization method or three-momentum cutoff $q_{\max }=1 \mathrm{GeV}$. However we can now fine tune slightly the subtraction constant or the cutoff to agree better with the experimental value of the axialvector resonances. Furthermore, as done in Ref. [14], we can also allow that the $f$ constant in Eq. (41) may be $f=115 \mathrm{MeV}$ instead of $92 \mathrm{MeV}$ in some cases due to the kaon and eta effects. Thus, in the present work we use the following values of the subtraction constant, $a$, and $f$ for the different channels: for $I=1, a=-1.95, f=92 \mathrm{MeV}$; for $I=0, G$-parity $+, a=-1.88, f=92 \mathrm{MeV}$; for $I=0$, $G$-parity -, $a=-0.80, f=115 \mathrm{MeV}$; and for $I=1 / 2$, $a=-1.85, f=115 \mathrm{MeV}$. It is important to emphasize that once this slight fine tune of the regularization pa-

\footnotetext{
2 Recall that the $G$-parity operation can be defined through its action on an eigenstate of hypercharge $(Y)$, isospin $(I)$, and third isospin projection $\left(I_{3}\right)$ as $G\left|Y, I, I_{3}\right\rangle=\eta(-1)^{Y / 2+I}\left|-Y, I, I_{3}\right\rangle$, with $\eta$ being the charge conjugation of a neutral non-strange member of the $S U(3)$ family.
}

rameters is done, the model discussed later in the present work for the three-body interaction will have no further freedom.
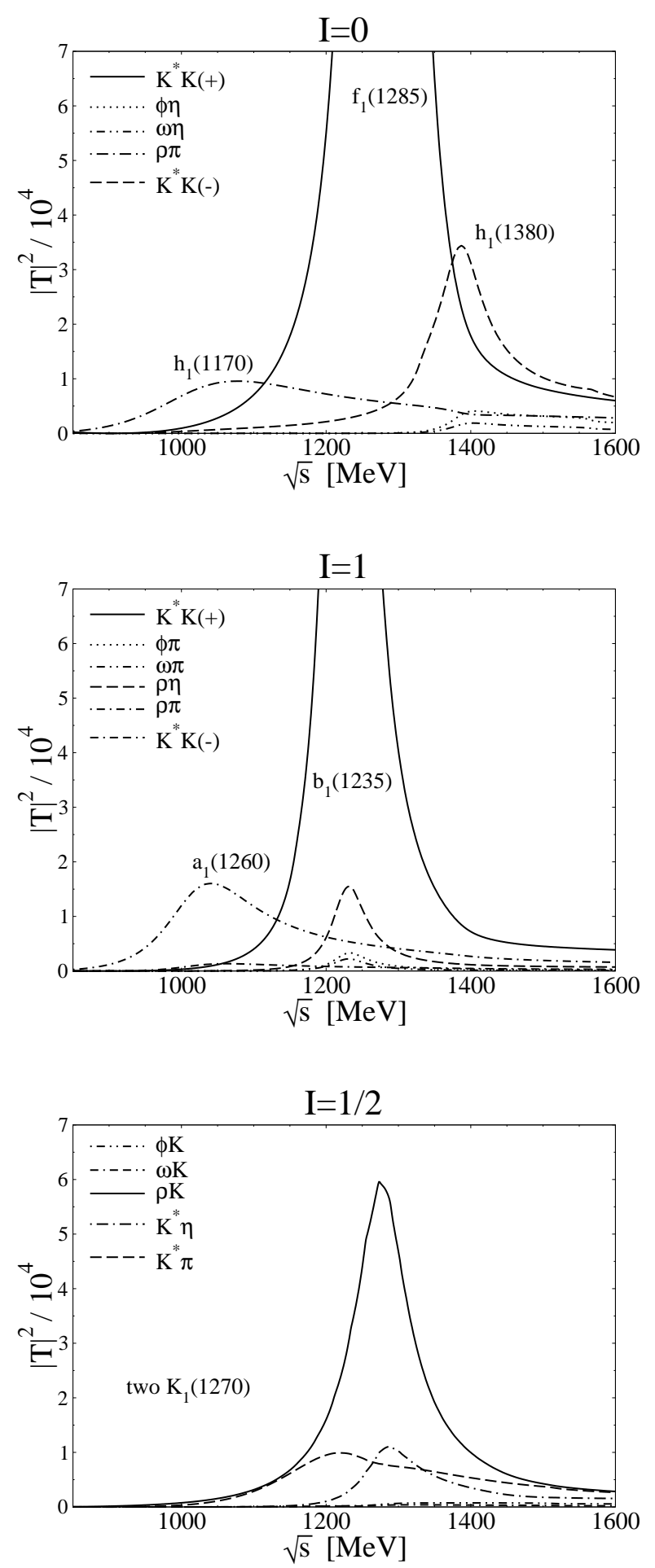

FIG. 3. Modulus squared of the pseudoscalar-vector unitarized scattering amplitudes for the different isospin channels. The labels indicate the correspondent experimental particles to which the resonances dynamically generated are associated. 
In Fig. 3 we show the modulus squared of some of the diagonal $P V$ unitarized amplitudes. Note that the plots differ slightly from those in Ref. [13] since we have used now slight different values of the subtraction constants and the loop function with dimensional regularization is now folded with the vector meson width, which was not done in Ref. [13].

It is worth stressing that the unitarized amplitudes provide not only the masses and widths of the dynamically generated resonances but also the actual shape of the scattering amplitude. Certainly that includes whatever background it could contain which would be also generated thanks to the highly non-linear dynamics involved in the unitarization procedure. Thus, even if there is no resonance in a particular channel the method provides the right amplitude for it.

\section{THREE-BODY INTERACTION}

In this section we explain the technical details for the evaluation of the three body interaction between one pseudoscalar particle and two vector mesons in spin two and s-wave. As seen in section II A, whenever we have two vector mesons with parallel spins they tend to bind very strongly. For instance, we have seen that two $\rho$ mesons in spin two and s-wave bind very strongly forming an $f_{2}(1270)$. This implies a binding energy per $\rho$ particle of about $140 \mathrm{MeV}$ which is almost $20 \%$ of the $\rho$ meson mass. Similar qualitative reasonings can be done for the other tensor mesons discussed in section \A, $\left(K_{2}^{*}(1430)\right.$ as a $K^{*} \rho$ cluster and $a_{2}(1320)$ as $\left.K^{*} \bar{K}^{*}\right)$. Therefore we can expect that inside the three body $P V V$ system with spin two the two vector mesons will be clustered forming a tensor meson. In such a case, we can apply the fixed center approximation to the Faddeev equations, which otherwise would be very difficult to solve exactly. As mentioned in the introduction, the FCA has been proved suited and good enough in similar three body systems when two of the particles tend to cluster together.

For the technicals details we follow closely the steps of Refs. [39, 40] with the proper adaptations and modifications to the present case. In what follows we are going to explain generically the interaction of a particle $A$ with a cluster $B$ made of two particles, $b_{1}$ and $b_{2}$. For the present work the particle $A$ will represent the pseudoscalar particle and $b_{1}, b_{2}$, the two vector mesons that build up the tensor cluster $B$. Specifically, we study the system $P V V$ with $J^{P C}=2^{-+}$with three different possible isospin, $I=1,0$, and $1 / 2$. The $V V$ clusters are the $2^{++}$tensor mesons $a_{2}(1320), f_{2}(1270)$ and $K_{2}^{*}(1430)$ and the pseudoscalars are $\pi, K$ and $\eta$. In the following we represent $a_{2}(1320), f_{2}(1270)$ and $K_{2}^{*}(1430)$ by $a_{2}, f_{2}$ and $K_{2}^{*}$ respectively.

The allowed channels for the different isospins, taking also into account the $G$-parity restrictions, are shown in table [. These are: for $I=1, \pi f_{2}$ and $\eta a_{2}$; for $I=0$, $\pi a_{2}$ and $\eta f_{2}$; and for $I=1 / 2, \pi K_{2}^{*}, K a_{2}, K f_{2}$ and $\eta K_{2}^{*}$.

\begin{tabular}{|c|c|c|c|c|}
\hline $\begin{array}{c}\text { total } P V V \\
\text { isospin }\end{array}$ & \multicolumn{4}{|c|}{ channels $A B\left(b_{1} b_{2}\right)$} \\
\hline$I=1$ & $\pi f_{2}(\rho \rho)$ & $\eta a_{2}\left(K^{*} \bar{K}^{*}\right)$ & & \\
\hline$I=0$ & $\pi a_{2}\left(K^{*} \bar{K}^{*}\right)$ & $\eta f_{2}(\rho \rho)$ & & \\
\hline$I=\frac{1}{2}$ & $\pi K_{2}^{*}\left(\rho K^{*}\right)$ & $K a_{2}\left(K^{*} \bar{K}^{*}\right)$ & $K f_{2}(\rho \rho)$ & $\eta K_{2}^{*}\left(\rho K^{*}\right)$ \\
\hline
\end{tabular}

TABLE I. Possible pseudoscalar-tensor channels for the different total $P V V$ isospins. The particles in parenthesis represent the main vector-vector component of the tensor resonances. The $a_{2}, f_{2}$ and $K_{2}^{*}$ symbols stand for $a_{2}(1320), f_{2}(1270)$ and $K_{2}^{*}(1430)$ respectively.

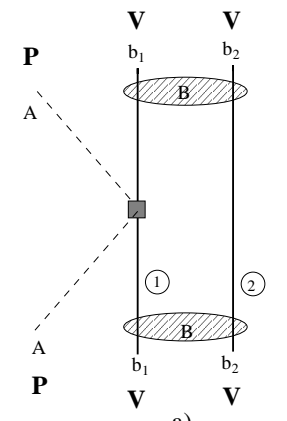

a)

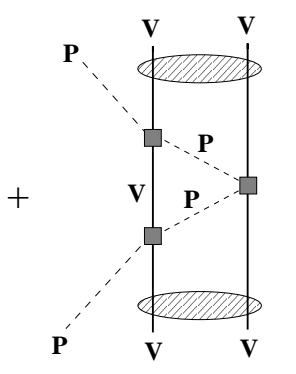

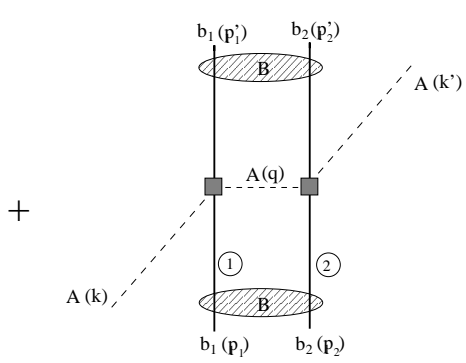

b)

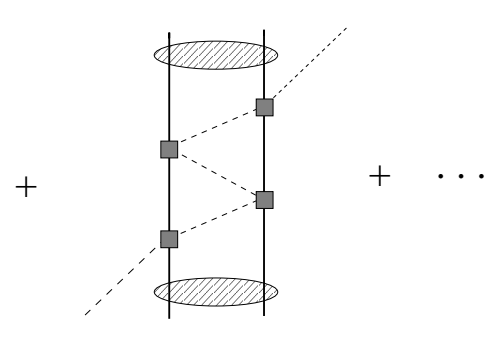

c)
FIG. 4. Diagrammatic representation of the fixed center approximation to the Faddeev equations for the interaction of a pseudoscalar particle, $A$, with a tensor particle, $B$, which is a cluster made of two vector mesons, $b_{1}$ and $b_{2}$. Diagrams $a$ ) and $b$ ) represent the single and double scattering contributions respectively and $b)+c$ ) the multiple scattering contribution.

Note that in principle for isospin $I=1$ the $K \bar{K}_{2}^{*}$ and $\bar{K} K_{2}^{*}$ are possible with the negative $G$-parity combination $1 / \sqrt{2}\left|K \bar{K}_{2}^{*}+\bar{K} K_{2}^{*}\right\rangle$, however, when doing later the $P V$ interaction to evaluate the three body amplitude, the possible configurations with these channels do not respect the total $G$-parity and are thus not allowed. A similar argument forbids the $1 / \sqrt{2}\left|K \bar{K}_{2}^{*}-\bar{K} K_{2}^{*}\right\rangle G$-parity $(+)$ channel in the $I=0$ case. (This is explained in further detail at the end of the Appendix).

The FCA to the Faddeev equations is represented diagrammatically in Fig. 4. The pseudoscalar particle, $A$, rescatters repeatedly with each of the vector mesons, $b_{i}$, which form the tensor resonance cluster, $B$. The thick squared dots in the figure represent the unitarized $P V$ 
interaction discussed in section IIB In the figure only the interaction starting with particle $b_{1}$ is represented, but an analogous mechanism where particle $A$ starts the interaction against particle $b_{2}$ must also be considered. Mathematically, the FCA can be written as a system of coupled equations

$$
\begin{aligned}
T_{1} & =t_{1}+t_{1} G_{0} T_{2} \\
T_{2} & =t_{2}+t_{2} G_{0} T_{1} \\
T & =T_{1}+T_{2}
\end{aligned}
$$

where $T_{1}, T_{2}$, are the two partition functions which sum up to the total scattering matrix, $T$. The $T_{i}$ amplitudes accounts for all the diagrams starting with the interaction of the particle $A$ with particle $b_{i}$ of the compound system $B$. In Eq. (8) $G_{0}$ is the Green function for the exchange of a particle $A$ between the $b_{1}$ and $b_{2}$ particles (intermediate dashed lines in Fig. (4) which expression will be given below, (see Eq. (19)). The mechanism in Fig. 4a represents the single-scattering contribution $\left(t_{1}\right.$ in Eq. (80) and Fig. 4b the double-scattering mechanism (the next contribution: $t_{1} G_{0} t_{2}$ ). The addition of Fig. 45 represents the full resummation of mechanisms to get the full $T_{1}$ partition function in the FCA. An analogous figure starting with the particle $A$ interacting with $b_{2}$ would account for the $T_{2}$ amplitude.

Note that the FCA equations, Eq. (8), are essentially given in terms of the two-body pseudoscalar-vector amplitudes, $t_{1}$ and $t_{2}$, for which we used the unitarized $P V$ amplitudes given in section IIB. The argument of the function $T(s)$ in Eq. (8) is the total invariant mass energy of the $P V V$ system, $s$. However the argument of $t_{1}$ and $t_{2}$ are $s_{1}$ and $s_{2}$, where $s_{i}(i=1,2)$ is the invariant mass of the interacting particle $A$ and the particle $b_{i}$ of the $B$ molecule and is given by

$s_{i}=m_{A}^{2}+m_{b_{i}}^{2}+\frac{1}{2 m_{B}^{2}}\left(s-m_{A}^{2}-m_{B}^{2}\right)\left(m_{B}^{2}+m_{b_{i}}^{2}-m_{b_{j \neq i}}^{2}\right)$,

where $m_{A(B)}$ is the mass of the $A(B)$ system and $m_{b_{i}}$ is the mass of each vector meson of the $B$ molecule.

The derivation of the expression of the single scattering amplitudes, $t_{i}$, in terms of the unitarized two body $P V$ amplitudes of section IIB is explained in detail in the Appendix.

Proceeding in an analogous way to the derivation done in Refs. [39, 40], properly adapted to the present problem, we can obtain the $S$ matrix for the single scattering contribution:

$$
S^{(1)}=S_{1}^{(1)}+S_{2}^{(1)},
$$

with

$$
\begin{aligned}
& S_{i}^{(1)}=-i t_{A b_{i}} \frac{1}{\mathcal{V}^{2}} \frac{1}{\sqrt{2 \omega_{p_{i}}}} \frac{1}{\sqrt{2 \omega_{p_{i}^{\prime}}}} \frac{1}{\sqrt{2 \omega_{k}}} \frac{1}{\sqrt{2 \omega_{k^{\prime}}}} \\
& \times(2 \pi)^{4} \delta\left(k+k_{B}-k^{\prime}-k_{B}^{\prime}\right) F_{B, i}\left(\frac{\mu}{m_{i}}\left(\vec{k}-\vec{k}^{\prime}\right)\right) .
\end{aligned}
$$

where $t_{A b_{i}}$ are the single scattering amplitudes given in the Appendix, $\mathcal{V}$ represents the volume of a box where we normalize to unity the plane wave states, the momenta are defined in Fig. 4 $\mathrm{b}, \omega_{p}$ represents the on-shell energy of the corresponding particle with momentum $p, k_{B}\left(k_{B}^{\prime}\right)$ represents the total momentum of the initial (final) cluster $B$ and $\mu$ is the reduced mass of the $b_{1} b_{2}$ system with masses $m_{1}, m_{2}$, respectively. In Eq. (11), $F_{B, i}$ is the form factor of the particle $B$ which represents essentially the Fourier transform of its wave function. The derivation of the form factor is similar to the one done in Refs. [39, 46], where we refer also for further discussion and interpretation. The form factor has to be projected onto s-wave, the one we are considering in the present work. Hence, $F_{B, i}$ in Eq. (11) is replaced by

$$
F_{B, i}^{(s)}(s)=\frac{1}{2} \int_{-1}^{1} d \cos \theta_{s} F_{B}(\vec{v})
$$

where $\vec{v} \equiv \frac{\mu}{m_{i}}\left(\vec{k}-\vec{k}^{\prime}\right)$ with $|\vec{v}|=\frac{\mu}{m_{i}} k \sqrt{2\left(1-\cos \theta_{s}\right)}$, $k=\sqrt{\left(s-\left(M_{A}+M_{B}\right)^{2}\right)\left(s-\left(M_{A}-M_{B}\right)^{2}\right)} / 2 \sqrt{s}$ above the $A B$ threshold and zero below. The $B$ resonance form factor, $F_{B}(\vec{v})$, is given by

$$
\begin{aligned}
F_{B}(\vec{v}) & =\frac{1}{\mathcal{N}} \int_{\substack{|\vec{p}|<\Lambda \\
|\vec{p}-\vec{v}|<\Lambda}} d^{3} p \frac{1}{m_{B}-\omega_{1}(\vec{p})-\omega_{2}(\vec{p})+i \frac{\Gamma_{1}+\Gamma_{2}}{2}} \\
& \times \frac{1}{m_{B}-\omega_{1}(\vec{p}-\vec{v})-\omega_{2}(\vec{p}-\vec{v})+i \frac{\Gamma_{1}+\Gamma_{2}}{2}}, \\
\mathcal{N} & =\int_{|\vec{p}|<\Lambda} d^{3} p \frac{1}{\left(m_{B}-\omega_{1}(\vec{p})-\omega_{2}(\vec{p})+i \frac{\Gamma_{1}+\Gamma_{2}}{2}\right)^{2}} .
\end{aligned}
$$

where $\omega_{j}(\vec{q})=\sqrt{\vec{q}^{2}+m_{j}^{2}}$ and $\Gamma_{j}$ is the width of the $b_{j}$ particle. In Eq. (13), $\Lambda$ represents a three-momentum cutoff with a similar physical meaning [39] as the threemomentum cutoff of the vector-vector loop function of Eq. (2). For that reason we take for $B=a_{2}, f_{2}$ and $K_{2}^{*}$ the same values for $\Lambda$ as for the cutoffs mentioned in section $\Pi A$ for the corresponding channels. The $1 / \mathcal{N}$ factor is introduced in order to normalize to unity the form factor at zero momentum.

In Fig. 5 we show the form factors for the $a_{2}, f_{2}$ and $K_{2}^{*}$ as a function of the modulus of the momentum.

The inclusion of the form factor in the single scattering contribution, Eq. (11), can be relevant for energies far above the $A B$ threshold. In the present work we are above threshold only in the channels where the particle $A$ is a pion. We have checked that, in any case, the numerical effect of this form factor in the single scattering mechanism is small. However for the multiple scattering mechanisms the form factor is a key ingredient as we shall see below.

The next order contribution of the FCA to the Faddeev equations is the double scattering mechanism, which corresponds to Fig. $4 \mathrm{~b}$. The $S$-matrix for this contribution takes the form

$$
S^{(2)}=S_{1}^{(2)}+S_{2}^{(2)},
$$




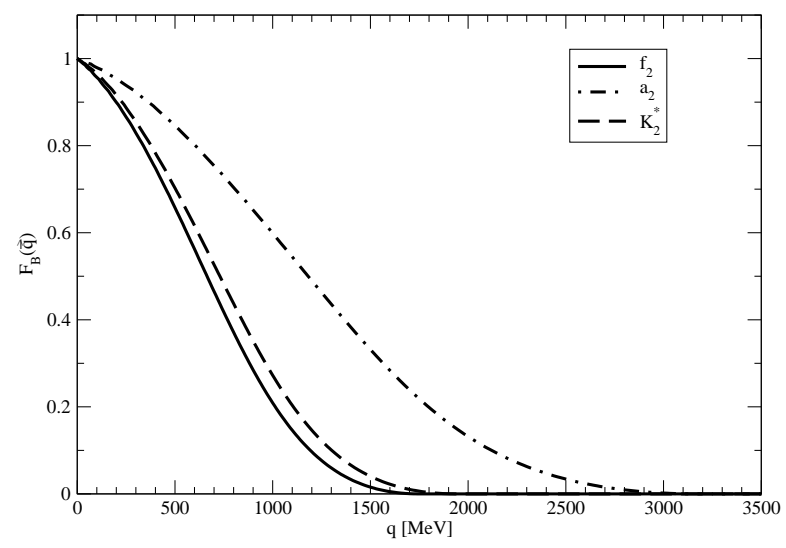

FIG. 5. Form factors for the $a_{2}, f_{2}$ and $K_{2}^{*}$ tensor mesons.

with

$S_{i}^{(2)}=-i(2 \pi)^{4} \delta\left(k+k_{B}-k^{\prime}-k_{B}^{\prime}\right) \frac{1}{\mathcal{V}^{2}} \frac{1}{\sqrt{2 \omega_{k}}} \frac{1}{\sqrt{2 \omega_{k^{\prime}}}}$

$\times \frac{1}{\sqrt{2 \omega_{p_{1}}}} \frac{1}{\sqrt{2 \omega_{p_{1}^{\prime}}}} \frac{1}{\sqrt{2 \omega_{p_{2}}}} \frac{1}{\sqrt{2 \omega_{p_{2}^{\prime}}}}$

$\times \int \frac{d^{3} q}{(2 \pi)^{3}} F_{B}\left(\vec{q}-\frac{\vec{k} m_{j \neq i}+\vec{k}^{\prime} m_{i}}{m_{1}+m_{2}}\right) \frac{1}{q^{0^{2}}-\vec{q}^{2}-m_{A}^{2}+i \epsilon}$

$\times t_{A b_{1}} t_{A b_{2}}$.

with $q^{0}(s ; A, B)=\left(s-m_{A}^{2}-m_{B}^{2}\right) /\left(2 m_{B}\right)$. The term $\frac{\vec{k} m_{j \neq i}+\vec{k}^{\prime} m_{i}}{m_{1}+m_{2}}$ inside the argument of the form factor is $m_{1}+m_{2}$
small for bound states, below threshold, and thus it is not considered in previous works regarding the FCA. If one considers this term, then $F_{B}$ must be projected onto s-wave as in Eq. (12). We have checked that the term $\frac{\vec{k} m_{j \neq i}+\vec{k}^{\prime} m_{i}}{m_{1}+m_{2}}$ inside the argument of the form factor has a small numerical effect and thus we have not considered it in the numerical evaluations for computational time reasons.

On the other hand, taking into account the general form of the $S$-matrix of an $A B$ interaction

$$
\begin{aligned}
S= & -i T(2 \pi)^{4} \delta\left(k+k_{B}-k^{\prime}-k_{B}^{\prime}\right) \frac{1}{\mathcal{V}^{2}} \\
& \times \frac{1}{\sqrt{2 \omega_{k}}} \frac{1}{\sqrt{2 \omega_{k^{\prime}}}} \frac{1}{\sqrt{2 \omega_{k_{B}}}} \frac{1}{\sqrt{2 \omega_{k_{B}^{\prime}}}} .
\end{aligned}
$$

and comparing this equation with Eqs. (11) and (15), we obtain that the FCA equations (8) take in our case the form

$$
\begin{aligned}
T_{A b_{1}} & =F_{B, 1}^{(s)} \bar{t}_{1}+\bar{t}_{1} G_{0} T_{A b_{2}} \\
T_{A b_{2}} & =F_{B, 2}^{(s)} \bar{t}_{2}+\bar{t}_{2} G_{0} T_{A b_{1}} \\
T & =T_{A b_{1}}+T_{A b_{2}}
\end{aligned}
$$

with

$$
\bar{t}_{i}=\sqrt{\frac{\omega_{k_{B}} \omega_{k_{B}^{\prime}}}{\omega_{p_{i}} \omega_{p_{i}^{\prime}}}} t_{A b_{i}}\left(s_{i}\right) .
$$

Note that the argument of the $t_{A b_{i}}$ function is $s_{i}$ of Eq. (91), whereas the total amplitude $T$ can be regarded as a function of the global $s$. In Eq. (17)

$$
\begin{aligned}
& G_{0}(s ; A, B)= \\
& \frac{1}{2 \sqrt{\omega_{k_{B}} \omega_{k_{B}^{\prime}}}} \int \frac{d^{3} q}{(2 \pi)^{3}} F_{B}(\vec{q}) \frac{1}{q^{0}(s ; A, B)^{2}-\vec{q}^{2}-m_{A}^{2}+i \epsilon} .
\end{aligned}
$$

As an example, we show in Fig. 6 the real and imaginary parts of the $G_{0}$ function for the channel $\eta a_{2}$. Close to the threshold it resembles the typical shape of the loop function of two mesons, in this case one $\eta$ and one $a_{2}$. For the other channels $G_{0}$ has similar qualitative shapes but with different thresholds.

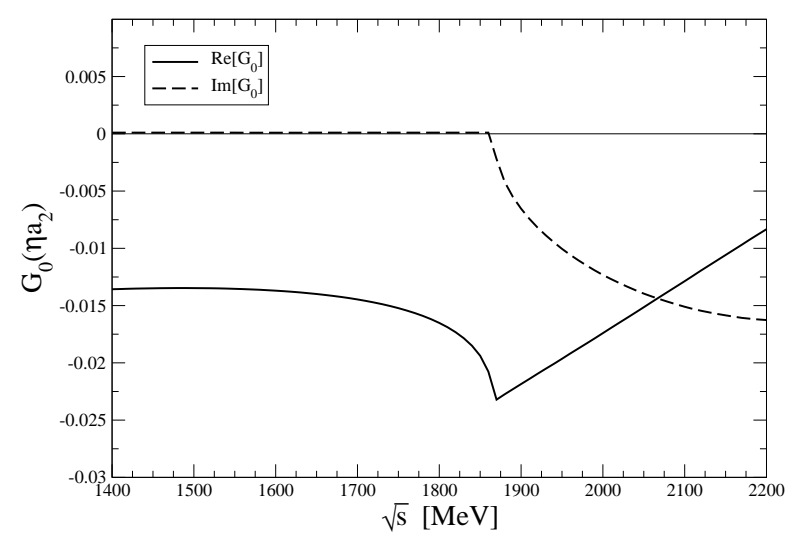

FIG. 6. $G_{0}$ function for the $\eta a_{2}$ channel.

Solving algebraically the system of equations (17) gives the following final three-body scattering amplitude

$$
\begin{aligned}
T= & T_{A b_{1}}+T_{A b_{2}}=\frac{\bar{t}_{1}+\bar{t}_{2}+2 \bar{t}_{1} \bar{t}_{2} G_{0}}{1-\bar{t}_{1} \bar{t}_{2} \widetilde{G}_{0}^{2}} \\
& +\bar{t}_{1}\left(F_{B, 1}^{(s)}-1\right)+\bar{t}_{2}\left(F_{B, 2}^{(s)}-1\right)
\end{aligned}
$$

Thus far we have not considered anywhere the finite width of the tensor resonance $B$. We have taken this effect into account by folding the final amplitude $T\left(M_{B}\right)$, regarded as a function of $M_{B}$, with the spectral function of the $B$ resonance:

$$
\begin{aligned}
& T \rightarrow T=\frac{1}{\mathcal{N}_{B}} \int_{\left(M_{B}-2 \Gamma_{B}^{0}\right)^{2}}^{\left(M_{B}+2 \Gamma_{B}^{0}\right)^{2}} d s_{B} \\
& \times \operatorname{Im}\left\{\frac{1}{s_{B}-M_{B}^{2}+i M_{B} \Gamma_{B}\left(s_{B}\right)}\right\} T\left(\sqrt{s_{V}}\right),
\end{aligned}
$$

$\mathcal{N}_{B}=\int_{\left(M_{B}-2 \Gamma_{B}^{0}\right)^{2}}^{\left(M_{B}+2 \Gamma_{B}^{0}\right)^{2}} d s_{B} \operatorname{Im}\left\{\frac{1}{s_{B}-M_{B}^{2}+i M_{B} \Gamma_{B}\left(s_{B}\right)}\right\}$.

where $\Gamma_{B}\left(s_{B}\right)$ is the energy dependent width of the $B$ particle and $\Gamma_{B}^{0}=\Gamma_{B}\left(M_{B}^{2}\right)$. 
Thus far we have only considered the interaction of one single channel consisting of one pseudoscalar meson and one tensor meson. We are going to estimate the possible coupled channels effect. If we look at table $\nabla$ in the Appendix, we see that for total isospin $I=1 / 2$ of the $P V V$ system we can have for single scattering interaction non diagonal scattering amplitudes. (This is not the case for $I=1$ and $I=0$ ). For instance, an initial $\pi K_{2}^{*}$ state can turn into $K a_{2}$ thanks to the transition $t_{\pi \rho, K \bar{K}^{*}}$. However a direct application of the FCA cannot be done in this case since the FCA requires that the $B$ cluster, the tensor meson in our case, is not much altered by the interaction with the $A$ particle, the pseudoscalar meson. Otherwise one should evaluate the mechanisms of the multistep processes with quantum field theory evaluating the corresponding three meson loops, etc. This will spoil the simplicity of the FCA approximation since the problem becomes very involved with the higher iterations and ultimately turns out into a problem far more complicated than the use of the exact Faddeev equations from the beginning. The wave functions used in the derivation of the S-matrix and the form factors (see Ref. [39]) are now different for the initial and final cluster $B$ and thus the derivation in this section is not directly valid. The differences are essentially due to the different masses between different channels. However, in the present case the constituent particles of the $B$ resonance of the initial and final state are all vector mesons, and thus they have a similar mass and so are the typical momenta inside the clusters $B$. On the other hand, doing coupled channels is in general relevant if the final amplitude for the different channels have a similar strength. However, advancing some results, this is not the case in the present work. From all this reasons we can conclude that the couple channel effects will be small and thus we can just estimate its effect adapting the formalism discussed so far.

The equation for the coupled channel estimation is now formally the same than Eq. (17) but now the amplitudes are $4 \times 4$ matrices (since we have four pseudoscalartensor channels for $I=1 / 2)$ such that $\left(T^{i}\right)_{j k}, i=1,2$; $j, k=1-4$, represents the interaction $T_{A_{j} b_{i, j} \rightarrow A_{k} b_{i, k}}^{i}$ of channel $j$ starting with particle $i$ of the cluster to produce channel $k$. Now $\left(\bar{t}^{i}\right)_{j k}=\sqrt{\frac{\omega_{B_{j}} \omega_{B_{k}}}{\omega_{i, j} \omega_{i, k}}} t_{A_{j} b_{i, j} \rightarrow A_{k} b_{i, k}}$. The $G_{0}$ function is now a diagonal $4 \times 4$ matrix which elements are $G_{0}\left(s ; A_{i}, B_{i}\right), i=1,4$. The form factor that multiplies the single scattering contribution in Eq. (17) is now a diagonal matrix which element $j j$ is $F_{B_{j}, i}^{(s)}$. Finally, to take into account the width of the $B$ particles, an independent folding with their spectral functions analogous to Eq. (21) is implemented for every different $B$ species.

\section{RESULTS}

In Fig. 7 we show the modulus squared of the threebody scattering amplitudes for each channel and for total
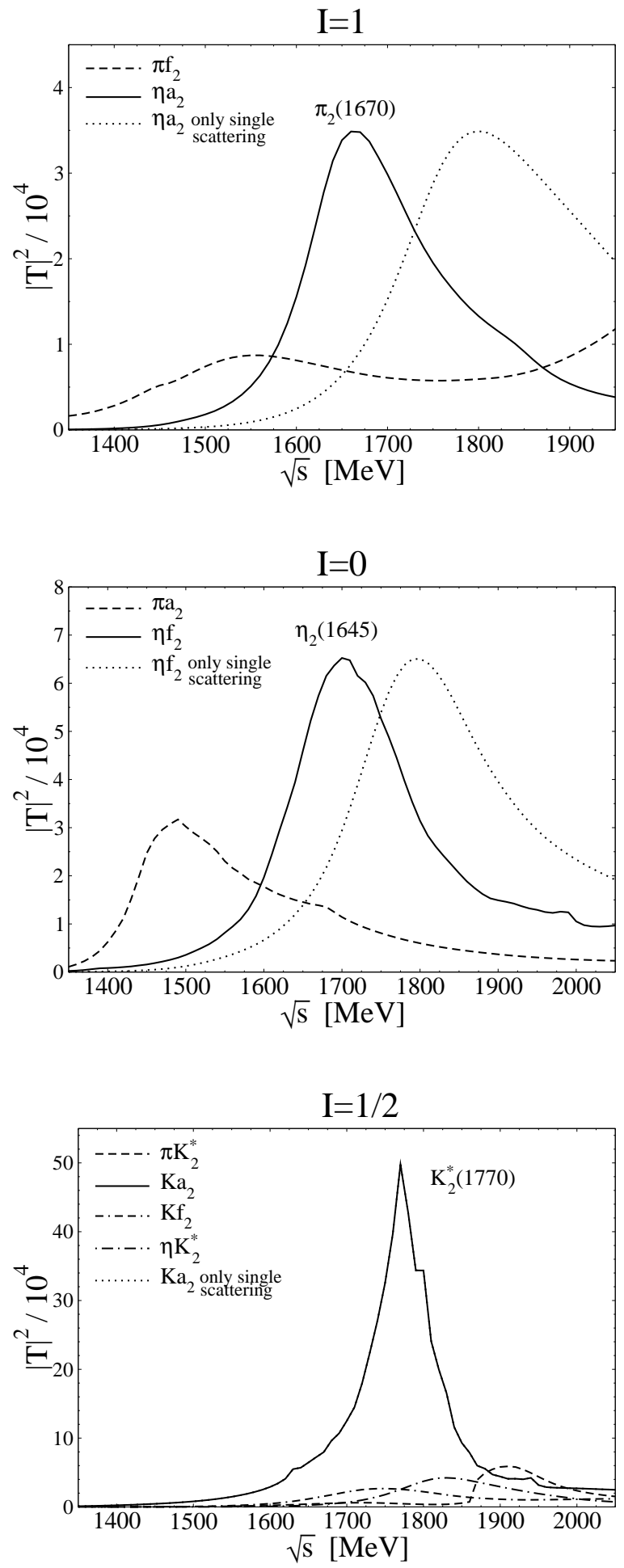

FIG. 7. Modulus squared of the three-body pseudoscalarvector-vector scattering amplitudes for the different total isospin channels. No coupled channels effect is considered for the $I=1 / 2$ case. The particle labels over the dominant peaks indicate the experimental pseudotensor $2^{-+}$mesons to which our dynamically generated are associated. 
isospin $I=1, I=0$ and $I=1 / 2$. The calculation accounts for the full model but without coupled channels in $I=1 / 2$, which will be discussed later on. In all the plots the dotted line represent the results for the dominant channels but considering only the single scattering contribution. The single scattering plots have been normalized to the peak of the dominant channel for every isospin in order to make easier the comparison of the position of the maxima (the real size of the dotted plots for $I=1$ and $I=0$ is actually about a factor three larger than the solid lines). In the $I=1 / 2$ case the dotted and solid lines overlap. The particle labels over the dominant peaks indicate the experimental pseudotensor mesons to which we associate our dynamically generated resonances.

Considering only the single scattering, resonant shapes are clearly visible in the plots but the position of the maxima do not agree well with the experimental value of the closest resonance in the corresponding channel, except for $I=1 / 2$. In table \II the value of the masses of the dynamically generated pseudotensor systems are shown in comparison with the experimental values at the PDG [47]. The second column of table [11 indicates the dominant channel, which is the one chosen to get the mass quoted in the table obtained from the position of the maximum.

When the multiple scattering mechanisms are added, an important improvement in the agreement with experimental masses is obtained for $I=1$ and $I=0$. For the $I=1 / 2$ channel no change is appreciable when adding the multiple scattering mechanisms but for this channel the mass obtained with single scattering contribution already agreed well with the experimental value for the $K_{2}^{*}(1770)$ resonance. The improvement obtained when considering the full mechanisms is an indication of the goodness and validity of the model proposed in the present work for the nature of the pseudotensor mesons considered. From the width of the amplitudes squared we can estimate the width of the dynamically generated pseudotensor states. We get for the $\pi_{2}(1670), \eta_{2}(1645)$ and $K_{2}^{*}(1770), 160 \mathrm{MeV}, 170 \mathrm{MeV}$ and $80 \mathrm{MeV}$ respectively, to be compared with the experimental values $260 \pm 9,181 \pm 11$ and $186 \pm 14$ respectively. The underestimation of the width within our model is not worrying. Indeed it is expected because a good reproduction of the width would imply to account also for other components and possible decay channels which can contribute to the decay width even if they do not significantly affect the mass. Therefore, in contrast with the mass results, which are quite reliable, our results for the width must be considered only qualitatively.

It is worth stressing the simplicity of our approach and that there are no free parameters in the model for the three-body scattering, once the regularization parameters of the $V V$ and $P V$ amplitudes are slightly changed to agree better with the tensor and axial-vector experimental masses respectively, as explained in section [II Therefore the results and conclusions of the present work are genuine predictions with no fits to any pseudotensor meson parameter or experimental data.

The results allow us to conclude that our threebody model generates dynamically the $\pi_{2}(1670)$ as a dominantly $\eta a_{2}$ molecule, the $\eta_{2}(1645)$ as $\eta f_{2}$ and the $K_{2}^{*}(1770)$ as $K a_{2}$. Certainly other Fock space components like quark-antiquark, two meson components (different to those considered in the present work), etc, are present in the pseudotensor resonances. However the fact that with the picture of a pseudoscalar-tensor molecule, with the tensor itself being two vector mesons, we get good agreement for the value of the masses reinforces the idea that this component is the dominant one in the wave function of these pseudotensor mesons. The extra components could only affect the total width of the resonances, as explained above.

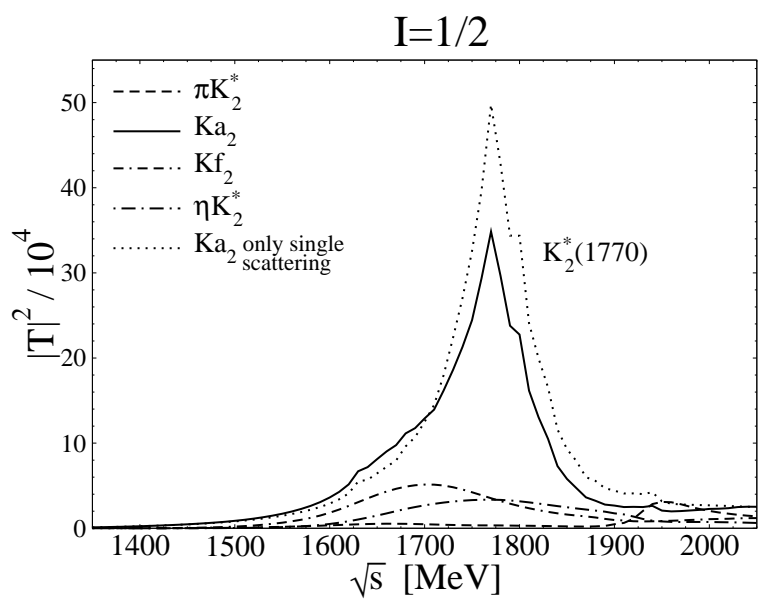

FIG. 8. Same meaning as in Fig. 7 for the $I=1 / 2$ case but implementing coupled channels.

In Fig. 8 we show the coupled channel effect in the $I=1 / 2$ channel. We see, by comparing with Fig. 7 that the effect is very small and in this particular case, since there is one channel so strongly dominant, $K a_{2}$, the coupled channel effects is negligible and thus there is no need to improve upon the estimation done in the present work.

\section{SUMMARY}

We have performed a theoretical study of the three body system consisting of one pseudoscalar and two vector mesons were the vector mesons are strongly correlated forming a tensor resonance. The motivation was that in previous works it was obtained that two vector mesons in spin 2 and s-wave tend to bind making up the lightest tensor mesons $2^{++}$and the interaction of a pseudoscalar and a vector meson in s-wave is also very attractive and generate dynamically the lightest axialvector resonances. Thus the $P V V$ system could be strongly attractive and generate $2^{-+}$resonant states. 


\begin{tabular}{|c|c|c|c|c|}
\hline $\begin{array}{c}\text { assigned } \\
\text { resonance }\end{array}$ & $\begin{array}{c}\text { dominant } \\
\text { channel }\end{array}$ & $\begin{array}{c}\text { mass } \\
\text { PDG [47] }\end{array}$ & $\begin{array}{c}\text { mass, only } \\
\text { single scatt. }\end{array}$ & $\begin{array}{c}\text { mass } \\
\text { full model }\end{array}$ \\
\hline$\pi_{2}(1670$ & $\eta a_{2}(1320)$ & $1672 \pm 3$ & 1800 & 1660 \\
\hline$\eta_{2}(1645)$ & $\eta f_{2}(1270)$ & $1617 \pm 5$ & 1795 & 1695 \\
\hline$K_{2}^{*}(1770)$ & $K a_{2}(1320)$ & $1773 \pm 8$ & 1775 & 1775 \\
\hline
\end{tabular}

TABLE II. Results for the masses of the dynamically generated pseudotensor resonances. (All units are MeV)

The three body amplitudes are evaluated solving the Faddeev equations in the fixed center approximation, which can be applied since two of the three particles are clustered. The three body amplitudes are written in terms of the unitarized $P V$ interactions, which are obtained from the application of the techniques of the chiral unitary approach. The method used for the threebody evaluation does not introduce any new parameter once the regularization in the $V P$ and $V V$ is chosen. This allows us to make genuine predictions which can be compared with experimental values for pseudotensor resonances.

In the three body amplitudes we obtain significant resonant signals which can be associated with the $\pi_{2}(1670)$, $\eta_{2}(1645)$ and $K_{2}^{*}(1770)$ experimental pseudotensor resonances, where the dominant channels in the making up of this resonances are $\eta a_{2}(1320), \eta f_{2}(1270)$ and $K a_{2}(1320)$ respectively.

In spite that other states like quark-antiquark, other meson-meson, several mesons, etc, can contribute to the wave functions of these pseudotensor resonances, the remarkable agreement obtained with our picture make us to consider the pseudoscalar-tensor mesons contribution as the dominant component in the building up of these resonances.

\section{ACKNOWLEDGMENTS}

We thank E. Oset for useful discussions. This work is partly supported by DGICYT contracts FIS200603438, FPA2010-17806, the Fundación Séneca grant 11871/PI/09 and the EU Integrated Infrastructure Initiative Hadron Physics Project under Grant Agreement n.227431.

\section{APPENDIX: SINGLE SCATTERING IN TERMS OF TWO-BODY AMPLITUDES}

We derive in this Appendix the exact expression of the single scattering amplitudes, $t_{i}$, in terms of the unitarized $P V$ scattering amplitudes. The latter are calculated for a given $P V$ isospin. Therefore, we need to write the total isospin state of the global system $A B,\left|I_{A}, I_{B}, I, M\right\rangle$, in terms of the coupled isospin state of particle $A$ and $b_{i}$, $\left|I_{A}, I_{i}, I_{A i}, M_{A i}\right\rangle \otimes\left|I_{j}, M_{j}\right\rangle:$

$$
\begin{aligned}
& \left|I_{A}, I_{B}, I, M\right\rangle^{(i)}= \\
& \sum_{I_{A i}} \sum_{M_{A i}} \sum_{M_{A}} \mathcal{C}\left(I_{A}, I_{B}, I \mid M_{A}, M-M_{A}, M\right) \\
& \times \mathcal{C}\left(I_{i}, I_{j}, I_{B} \mid M_{A i}-M_{A}, M-M_{A i}, M-M_{A}\right) \\
& \times \mathcal{C}\left(I_{A}, I_{i}, I_{A i} \mid M_{A}, M_{A i}-M_{A}, M_{A i}\right)\left[\eta-\delta_{i 1}(\eta-1)\right] \\
& \times\left|I_{A}, I_{i}, I_{A i}, M_{A i}\right\rangle \otimes\left|I_{j}, M-M_{A i}\right\rangle
\end{aligned}
$$

where the $i$ label means that we are correlating the particle $A$ with particle $b_{i}, i=1,2, j \neq i, I_{A}$ is the isospin of the particle $A, I_{B}$ the isospin of particle $B$, $I$ the total $A B$ isospin, $I_{i}$ the isospin of particle $b_{i}, I_{A i}$ the global isospin of the $A-b_{i}$ system, and the $M_{x}$ are the third components of the corresponding isospins. In Eq. (22), $\eta=(-1)^{I_{1}+I_{2}-I_{B}}, \delta$ is the Kronecker delta and $\mathcal{C}\left(j_{1}, j_{2}, j_{3} \mid m_{1}, m_{2}, m_{3}\right)$ represent Clebsch-Gordan coefficients. For example, for total isospin $I=0$, one of the possible channels is $\pi a_{2}$, with $A=\pi, B=a_{2}, b_{1}=K^{*}$ and $b_{2}=\bar{K}^{*}$. In this case for the $A b_{1}$ interaction we have

$$
\begin{aligned}
\left(\pi a_{2}\right)_{I=0, M=0}^{(1)}= & -\frac{1}{\sqrt{2}}\left(\pi K^{*}\right)_{1 / 2,-1 / 2} \bar{K}^{* 0} \\
& -\frac{1}{\sqrt{2}}\left(\pi K^{*}\right)_{1 / 2,+1 / 2} K^{*-} .
\end{aligned}
$$

Throughout the work we have used the following isospin conventions: $\left|\pi^{+}\right\rangle=-|1,+1\rangle,\left|\rho^{+}\right\rangle=-|1,+1\rangle,\left|a_{2}^{+}\right\rangle=$ $-|1,+1\rangle,\left|K^{-}\right\rangle=-\left|\frac{1}{2},-\frac{1}{2}\right\rangle,\left|K^{*-}\right\rangle=-\left|\frac{1}{2},-\frac{1}{2}\right\rangle,\left|K_{2}^{*-}\right\rangle=$ $-\left|\frac{1}{2},-\frac{1}{2}\right\rangle$ (for the other particles the sign is positive) as is usually used in chiral perturbation theory and in the work from which our $P V$ amplitudes is based [13].

The scattering potential for the single scattering contribution can be written in terms of the two body amplitudes, $t_{A b_{i}, A^{\prime} b_{j^{\prime}}}$, for the transition $A b_{i} \rightarrow A^{\prime} b_{j^{\prime}}$ : 


$$
\begin{aligned}
& { }^{(i)}\left\langle A b_{1} b_{2}|V| A^{\prime} b_{1}^{\prime} b_{2}^{\prime}\right\rangle^{\left(i^{\prime}\right)}=\sum_{I_{A i}}\left[\sum_{M_{A i}} \sum_{M_{A}} \sum_{M_{A}^{\prime}}\right. \\
& \times \mathcal{C}\left(I_{A}, I_{B}, I \mid M_{A}, M-M_{A}, M\right) \\
& \times \mathcal{C}\left(I_{A^{\prime}}, I_{B^{\prime}}, I \mid M_{A^{\prime}}, M-M_{A^{\prime}}, M\right) \\
& \times \mathcal{C}\left(I_{i}, I_{j}, I_{B} \mid M_{A i}-M_{A}, M-M_{A i}, M-M_{A}\right) \\
& \times \mathcal{C}\left(I_{i^{\prime}}, I_{j^{\prime}}, I_{B^{\prime}} \mid M_{A i^{\prime}}-M_{A}, M-M_{A i^{\prime}}, M-M_{A^{\prime}}\right) \\
& \times \mathcal{C}\left(I_{A}, I_{i}, I_{A i} \mid M_{A}, M_{A i}-M_{A}, M_{A i}\right) \\
& \left.\times \mathcal{C}\left(I_{A^{\prime}}, I_{i^{\prime}}, I_{A i} \mid M_{A^{\prime}}, M_{A i}-M_{A^{\prime}}, M_{A i}\right)\right] \\
& \times\left[\eta-\delta_{i 1}(\eta-1)\right]\left[\eta^{\prime}-\delta_{i^{\prime} 1}\left(\eta^{\prime}-1\right)\right] t_{A b_{i}, A^{\prime} b_{i^{\prime}}}^{\left(I_{A i}\right)} \\
& \equiv \sum_{I_{A i}} \alpha_{i, i^{\prime}} t_{A b_{i}, A^{\prime} b_{i^{\prime}}}^{\left(I_{A i}\right)}
\end{aligned}
$$

Note that only the diagonal $t_{A b_{i}, A b_{i}}$ is needed if not doing coupled channels. The expression in Eq. (24) is very convenient and easily implementable for computer evaluation and is general for any three-body system made of $A, b_{1}$ and $b_{2}$ and thus can be useful for other works where the FCA is applied. In tables III IV and V we show the two-body amplitudes obtained from Eq. (24) for the three different total $P V V$ isospins. The different $P V V$ channels are labeled by the pseudoscalar meson, the tensor resonance and in brackets the two vector mesons dominant in the formation of the tensor meson. For $I=1$ and $I=0$ the non-diagonal terms are zero and thus what we show in the table represent diagonal elements. For $I=1 / 2$ the first column represent the initial channel and the first row the final one. The non-diagonal terms are only needed in the estimation of the coupled channels.

\begin{tabular}{|c|c|c|}
\hline & $\pi f_{2}(\rho \rho)$ & $\eta a_{2}\left(K^{*} \bar{K}^{*}\right)$ \\
\hline$t_{1}$ & $\frac{2}{9} t_{\pi \rho, \pi \rho}^{(I=0)}+\frac{2}{3} t_{\pi \rho, \pi \rho}^{(I=1)}+\frac{10}{9} t_{\pi \rho, \pi \rho}^{(I=2)}$ & $t_{\eta K^{*}, \eta K^{*}}^{(I=1 / 2)}$ \\
\hline$t_{2}$ & $\frac{2}{9} t_{\pi \rho, \pi \rho}^{(I=0)}+\frac{2}{3} t_{\pi \rho, \pi \rho}^{(I=1)}+\frac{10}{9} t_{\pi \rho, \pi \rho}^{(I=2)}$ & $t_{\eta K^{*}, \eta K^{*}}^{(I=1 / 2)}$ \\
\hline
\end{tabular}

TABLE III. Three body single scattering amplitudes in terms of the unitarized two-body $(P V)$ amplitudes for total isospin $I=1$

\begin{tabular}{|l|c|c|}
\hline & $\pi a_{2}\left(K^{*} \bar{K}^{*}\right)$ & $\eta f_{2}(\rho \rho)$ \\
\hline$t_{1}$ & $t_{\pi K^{*}, \pi K^{*}}^{(I=1 / 2)}$ & $2 t_{\eta \rho, \eta \rho}^{(I=1)}$ \\
\hline$t_{2}$ & $t_{\pi K^{*}, \pi K^{*}}^{(I=1 / 2)}$ & $2 t_{\eta \rho, \eta \rho}^{(I=1)}$ \\
\hline
\end{tabular}

TABLE IV. Three body single scattering amplitudes in terms of the unitarized two-body $(P V)$ amplitudes for total isospin $I=0$

At this point let us explicit further the explanation why the $K \bar{K}_{2}^{*}$ and $\bar{K} K_{2}^{*}$ channels do not contribute to the $I=1$ and $I=0$ three-body amplitudes. We will discuss the $I=1$ case since the $I=0$ reasoning is totally analogous. The channel with $I=1$ that we are considering has negative $G$-parity. The $K \bar{K}_{2}^{*}$ and $\bar{K} K_{2}^{*}$ states do not have defined $G$-parity by themselves. However if we define $K K_{2}^{*}( \pm) \equiv 1 / \sqrt{2}\left|K \bar{K}_{2}^{*} \mp \bar{K} K_{2}^{*}\right\rangle$, it is eigenstate of $G$ with eigenvalue \pm 1 . Thus in principle we should include $K K_{2}^{*}(-)$ as another channel in the global $I=1$ case. Let us consider first the $\left|K \bar{K}_{2}^{*}\right\rangle$ channel in $I=1$, $M=+1$. For the evaluations needed in the present work, we take the $K_{2}^{*}$ as a $\rho K^{*}$ state and for the evaluation of the FCA we need the interaction of the $K$ with the $\rho$ and the $\bar{K}^{*}$. Let us consider first the interaction of the kaon with the $\bar{K}^{*}$. Applying Eq. (22) we have

$$
\begin{aligned}
& \left(K \bar{K}_{2}^{*}\right)_{1,+1} \longrightarrow \\
& -\frac{1}{\sqrt{3}}\left[\left(K \bar{K}^{*}\right)_{0,0} \rho^{+}-\left(K \bar{K}^{*}\right)_{1,0} \rho^{+}-\left(K \bar{K}^{*}\right)_{1,+1} \rho^{0}\right], \\
& \left(\bar{K} K_{2}^{*}\right)_{1,+1} \longrightarrow \\
& -\frac{1}{\sqrt{3}}\left[\left(\bar{K} K^{*}\right)_{0,0} \rho^{+}+\left(\bar{K} K^{*}\right)_{1,0} \rho^{+}+\left(\bar{K} K^{*}\right)_{1,+1} \rho^{0}\right](25)
\end{aligned}
$$

Therefore we have to do

$$
\begin{aligned}
K K_{2}^{*}(-)= & \frac{1}{\sqrt{2}}\left(K \bar{K}_{2}^{*}+\bar{K} K_{2}^{*}\right)_{1,+1} \\
\longrightarrow \quad & -\frac{1}{\sqrt{3}}\left[\frac{1}{\sqrt{2}}\left(K \bar{K}^{*}+\bar{K} K^{*}\right)_{0,0} \rho^{+}\right. \\
& -\frac{1}{\sqrt{2}}\left(K \bar{K}^{*}+\bar{K} K^{*}\right)_{1,0} \rho^{+} \\
& \left.-\frac{1}{\sqrt{2}}\left(K \bar{K}^{*}+\bar{K} K^{*}\right)_{1,+1} \rho^{0}\right] .
\end{aligned}
$$

But $\frac{1}{\sqrt{2}}\left(K \bar{K}^{*}+\bar{K} K^{*}\right)$ is eigenstate of $G$ with eigenvalue +1 and, on the other hand, $\rho$ has $G$-parity $(+)$. Therefore under a $G$-parity transformation the right member of Eq. (26) has $G$-parity $(+)$ which is of opposite sign to what is required by the left term of Eq. (26). Therefore this channels is not permitted in the evaluation of the FCA of the Faddeev equations. An analogous reasoning leads to the same conclusion for the interaction of the kaon with the $\rho$ and also for the interaction needed in the global $I=0$ channel.
[1] N. Kaiser, P. B. Siegel and W. Weise, Phys. Lett. B 362, 23 (1995).

[2] J. A. Oller and E. Oset, Nucl. Phys. A 620 (1997) 438 [Erratum-ibid. A 652 (1999) 407].
[3] J.A. Oller, E. Oset and J. R. Peláez, Phys. Rev. Lett. 80 (1998) 3452; ibid, Phys. Rev. D 59 (1999) 74001.

[4] J. A. Oller and E. Oset, Phys. Rev. D 60 (1999) 074023.

[5] N. Kaiser, Eur. Phys. J. A 3, 307 (1998). 


\begin{tabular}{|c|c|c|c|c|}
\hline & $\pi K_{2}^{*}\left(\rho K^{*}\right)$ & $K a_{2}\left(K^{*} \bar{K}^{*}\right)$ & $K f_{2}(\rho \rho)$ & $\eta K_{2}^{*}\left(\rho K^{*}\right)$ \\
\hline$\pi K_{2}^{*}\left(\rho K^{*}\right)$ & $\begin{aligned} t_{1} & =\frac{1}{3} t_{\pi \rho, \pi \rho}^{(I=0)}+\frac{2}{3} t_{\pi \rho, \pi \rho}^{(I=1)} \\
t_{2} & =\frac{1}{9} t_{\pi K^{*}, \pi K^{*}}^{(I=1 / 2)}+\frac{8}{9} t_{\pi K^{*}, \pi K^{*}}^{(I=3 / 2)}\end{aligned}$ & $\begin{aligned} t_{1} & =-\frac{1}{2} t_{\pi \rho, K \bar{K}^{*}}^{(I=0)}+\frac{1}{\sqrt{6}} t_{\pi \rho, K \bar{K}}^{(I=1)} \\
t_{2} & =0\end{aligned}$ & $\begin{array}{l}t_{1}=0 \\
t_{2}=-\frac{2}{3 \sqrt{3}} t_{\pi K^{*}, K \rho}^{(I=1 / 2)}-\frac{8}{3 \sqrt{3}} t_{\pi K^{*}, K \rho}^{(I=3 / 2)}\end{array}$ & $\begin{aligned} t_{1} & =\sqrt{\frac{2}{3}} t_{\eta \rho, \eta \rho}^{(I=1)} \\
t_{2} & =-\frac{1}{3} t_{\pi K^{*}, \eta K^{*}}^{(I=1 / 2)}\end{aligned}$ \\
\hline$K a_{2}\left(K^{*} \bar{K}^{*}\right)$ & $\begin{aligned} t_{1} & =0 \\
t_{2} & =-\frac{1}{2} t_{K \bar{K}^{*}, \pi \rho}^{(I=0)}+\frac{1}{\sqrt{6}} t_{K \bar{K}^{*}, \pi \rho}^{(I=1)}\end{aligned}$ & $\begin{aligned} t_{1} & =0 \\
t_{2} & =\frac{3}{4} t_{K \bar{K}^{*}, K \bar{K}^{*}}^{(I=0)}+\frac{1}{4} t_{K}^{(I=1)} \bar{K}^{*}, K \bar{K}^{*}\end{aligned}$ & $\begin{aligned} t_{1} & =0 \\
t_{2} & =0\end{aligned}$ & $\begin{aligned} t_{1} & =0 \\
t_{2} & =\frac{1}{2} t_{K}^{(I=1)} \bar{K}^{*}, \eta \rho\end{aligned}$ \\
\hline$K f_{2}(\rho \rho)$ & $\begin{aligned} t_{1} & =-\frac{1}{3 \sqrt{3}} t_{K \rho, \pi K^{*}}^{(I=1 / 2)}-\frac{4}{3 \sqrt{3}} t_{K \rho, \pi K^{*}}^{(I=3 / 2)} \\
t_{2} & =-\frac{1}{3 \sqrt{3}} t_{K \rho, \pi K^{*}}^{(I=1 / 2)}-\frac{4}{3 \sqrt{3}} t_{K \rho, \pi K^{*}}^{(I=3 / 2)}\end{aligned}$ & $\begin{array}{l}t_{1}=0 \\
t_{2}=0\end{array}$ & $\begin{array}{l}t_{1}=\frac{2}{3} t_{K \rho, K \rho}^{(I=1 / 2)}+\frac{4}{3} t_{K \rho, K \rho}^{(I=3 / 2)} \\
t_{2}=\frac{2}{3} t_{K \rho, K \rho}^{(I=1 / 2)}+\frac{4}{3} t_{K \rho, K \rho}^{(I=3 / 2)}\end{array}$ & $\begin{aligned} t_{1} & =\frac{1}{\sqrt{3}} t_{K \rho, \eta K^{*}}^{(I=1 / 2)} \\
t_{2} & =\frac{1}{\sqrt{3}} t_{K \rho, \eta K^{*}}^{(I=1 / 2)}\end{aligned}$ \\
\hline$\eta K_{2}^{*}\left(\rho K^{*}\right)$ & $\begin{array}{l}t_{1}=\sqrt{\frac{2}{3}} t_{\eta \rho, \pi \rho}^{(I=1)} \\
t_{2}=-\frac{1}{3} t_{\eta K^{*}, \pi K^{*}}^{(I=1 / 2)}\end{array}$ & $\begin{aligned} t_{1} & =\frac{1}{2} t_{K \eta \rho, K \bar{K}^{*}}^{(I=1)} \\
t_{2} & =0\end{aligned}$ & $\begin{aligned} t_{1} & =0 \\
t_{2} & =\frac{2}{\sqrt{3}} t_{\eta K^{*}, K \rho}^{(I=1 / 2)}\end{aligned}$ & $\begin{array}{l}t_{1}=t_{\eta \rho, \eta \rho}^{(I=1)} \\
t_{2}=t_{\eta K^{*}, \eta K^{*}}^{(I=1 / 2)}\end{array}$ \\
\hline
\end{tabular}

TABLE V. Three body single scattering amplitudes in terms of the unitarized two-body $(P V)$ amplitudes for total isospin $I=1 / 2$

[6] E. Oset and A. Ramos, Nucl. Phys. A 635 (1998) 99.

[7] J. Nieves and E. Ruiz Arriola, Nucl. Phys. A 679, 57 (2000).

[8] J. A. Oller and U. G. Meissner, Phys. Lett. B 500, 263 (2001).

[9] C. Garcia-Recio, J. Nieves, E. Ruiz Arriola and M. J. Vicente Vacas, Phys. Rev. D 67, 076009 (2003).

[10] T. Hyodo, S. I. Nam, D. Jido and A. Hosaka, Phys. Rev. C 68, 018201 (2003).

[11] D. Jido, J. A. Oller, E. Oset, A. Ramos, U. G. Meissner, Nucl. Phys. A725 (2003) 181-200.

[12] M. F. M. Lutz, E. E. Kolomeitsev, Nucl. Phys. A730 (2004) 392-416.

[13] L. Roca, E. Oset, J. Singh, Phys. Rev. D72 (2005) 014002.

[14] L. S. Geng, E. Oset, L. Roca, J. A. Oller, Phys. Rev. D75 (2007) 014017.

[15] H. Nagahiro, K. Nawa, S. Ozaki, D. Jido and A. Hosaka, Phys. Rev. D 83 (2011) 111504.

[16] R. Molina, D. Nicmorus and E. Oset, Phys. Rev. D 78 (2008) 114018.

[17] L. S. Geng and E. Oset, Phys. Rev. D 79, 074009 (2009).

[18] R. Molina, H. Nagahiro, A. Hosaka and E. Oset, Phys. Rev. D 80, 014025 (2009).

[19] M. Bando, T. Kugo, S. Uehara, K. Yamawaki and T. Yanagida, Phys. Rev. Lett. 54 (1985) 1215.

[20] M. Bando, T. Kugo and K. Yamawaki, Phys. Rept. 164 (1988) 217.

[21] M. Harada and K. Yamawaki, Phys. Rept. 381, 1 (2003).

[22] U. G. Meissner, Phys. Rept. 161, 213 (1988).

[23] R. Molina, H. Nagahiro, A. Hosaka, E. Oset, Phys. Rev. D83 (2011) 094030.

[24] Y. Nogami Phys. Lett. 7 (1963) 288

[25] Y. Ikeda, T. Sato, Phys. Rev. C76 (2007) 035203.

[26] A. Martinez Torres, K. P. Khemchandani and E. Oset, Phys. Rev. C 77 (2008) 042203.

[27] D. Jido, Y. Kanada-En'yo, Phys. Rev. C78 (2008) 035203.
[28] G. Mennessier, J. Y. Pasquier, R. Pasquier, Phys. Rev. D6 (1972) 1351-1372.

[29] A. Martinez Torres, K. P. Khemchandani, L. S. Geng, M. Napsuciale and E. Oset, Phys. Rev. D 78 (2008) 074031.

[30] A. Martinez Torres, K. P. Khemchandani, D. Gamermann and E. Oset, Phys. Rev. D 80 (2009) 094012.

[31] L. D. Faddeev, Sov. Phys. JETP 12 (1961) 1014 [Zh. Eksp. Teor. Fiz. 39 (1960) 1459].

[32] M. Albaladejo, J. A. Oller, L. Roca, Phys. Rev. D82 (2010) 094019.

[33] R. Chand and R. H. Dalitz, Annals Phys. 20, 1 (1962)

[34] R. C. Barrett and A. Deloff, Phys. Rev. C 60, 025201 (1999).

[35] A. Deloff, Phys. Rev. C 61, 024004 (2000).

[36] S. S. Kamalov, E. Oset and A. Ramos, Nucl. Phys. A 690, 494 (2001).

[37] A. Gal, Int. J. Mod. Phys. A 22 (2007) 226.

[38] A. Martinez Torres, E. J. Garzon, E. Oset, L. R. Dai, Phys. Rev. D83 (2011) 116002.

[39] L. Roca and E. Oset, Phys. Rev. D 82, 054013 (2010).

[40] J. Yamagata-Sekihara, L. Roca, E. Oset, Phys. Rev. D82 (2010) 094017.

[41] J. -J. Xie, A. Martinez Torres, E. Oset, P. Gonzalez, Phys. Rev. C83 (2011) 055204.

[42] C. W. Xiao, M. Bayar, E. Oset, arXiv:1106.0459 [hep$\mathrm{ph}]$.

[43] M. Bayar, J. Yamagata-Sekihara, E. Oset, Phys. Rev. C84 (2011) 015209.

[44] S. Weinberg, Phys. Rev. 166 (1968) 1568; S. Coleman, J. Wess and B. Zumino, Phys. Rev. 177 (1969) 2239; C.G. Callan, S. Coleman, J. Wess and B. Zumino, ibid. 2247.

[45] M.C. Birse, Z. Phys. A 355 (1996) 231.

[46] J. Yamagata-Sekihara, J. Nieves and E. Oset, Phys. Rev. D 83 (2011) 014003.

[47] K. Nakamura et al. [Particle Data Group Collaboration], J. Phys. G G37 (2010) 075021. 